\title{
Speckle Tracking and Interferometric Processing of TerraSAR-X TOPS Data for Mapping Nonstationary Scenarios
}

\author{
Rolf Scheiber, Marc Jäger, Pau Prats-Iraola, Senior Member, IEEE, Francesco De Zan, and Dirk Geudtner
}

\begin{abstract}
Terrain observation by progressive scan (TOPS) antenna beam steering is utilized for European Space Agency's (ESA's) Sentinel-1 synthetic aperture radar (SAR) sensor for the interferometric wide swath (IW) and extra wide swath (EW) modes. As a consequence of the azimuth steering, the resulting signal characteristics have to be accounted for in SAR interferometric (InSAR) processing. This paper assesses the performance of speckle tracking and spectral diversity (SD) [also referred to as split spectrum or multi-aperture interferometry (MAI)] when applied to TOPS data acquired over nonstationary scenarios, such as glaciers. The characteristics of the TOPS signal, especially the azimuth-variant Doppler centroid, are discussed with particular consideration of along-track surface motion between the interferometric acquisitions. The TOPS specific coregistration requirements are formulated, followed by an analysis of the theoretical estimation accuracy as a function of the estimation window size. A refined adaptive coregistration approach based on SD is suggested. Experimental TerraSAR-X TOPS data acquired over the Lambert glacier, Antarctica, are used to validate the proposed speckle tracking and SD methodologies.
\end{abstract}

Index Terms-Burst-mode acquisitions, coregistration, Sentinel-11, synthetic aperture radar (SAR), terrain observation by progressive scan (TOPS), TerraSAR-X, wide-swath SAR modes.

\section{INTRODUCTION}

I N THE LAST two decades, synthetic aperture radar (SAR) interferometry has matured to become a powerful tool for assessing small changes on sub-wavelength scale over large areas of Earth's surface. Differential evaluations of the interferometric phase nowadays enable a unique range of applications including the measurement of subsidence due to ground water extraction or prospection, seismic displacements or glacier velocity measurements [1]. Prominent examples include the mapping of glacier flow of complete Antarctica [2] or the Bam earthquake [3].

The launch of European Space Agency's (ESA's) Sentinel-1A on April 3, 2014 entails the, to-date, unique opportunity to carry out routine, interferometric SAR-based observations of large

Manuscript received June 06, 2014; revised August 21, 2014; accepted September 18, 2014. This work was supported in part by the European Space Agency under contract 4000106648/12/NL/MP.

R. Scheiber, M. Jäger, P. Prats-Iraola, and F. De Zan are with the Microwaves and Radar Institute of the German Aerospace Center (DLR), 82234 Oberpfaffenhofen, Germany (e-mail: rolf.scheiber@dlr.de).

D. Geudtner is with the European Space and Technology Center of the European Space Agency (ESA), 2201 AZ Noordwijk, The Netherlands.

Color versions of one or more of the figures in this paper are available online at http://ieeexplore.ieee.org.

Digital Object Identifier 10.1109/JSTARS.2014.2360237 areas. Terrain observation by progressive scan (TOPS) antenna beam steering is utilized for the interferometric wide swath (IW) and extra wide swath (EW) modes to provide large swath width of $250 \mathrm{~km}$ at $5 \mathrm{~m} \times 20 \mathrm{~m}$ and $400 \mathrm{~km}$ at $20 \mathrm{~m} \times 40 \mathrm{~m}$ resolution, respectively [4]. The TOPS mode is designed to provide enhanced imaging performance in terms of SNR and azimuth ambiguity levels when compared with the conventional ScanSAR mode [5]. The suitability of TOPS data for interferometry was first demonstrated with experimental TOPS data acquired by TerraSAR-X for measuring subsidence in Mexico city. In this context, solutions for the critical coregistration step were developed for the first time [6]. This scenario considers vertical motion only, which is typical of subsidence and inflation and which will be referred to as stationary in the following. However, the more general case should additionally consider the horizontal surface motion, the along-track component being the critical one in TOPS acquisitions. We will refer to this case as nonstationary scenario. Typical examples include glacier flow and horizontal displacements caused by seismic events.

The most widely used offset tracking technique for interferometric SAR image pairs is based on cross correlation applied to detected data, as described in detail for example in [7]. The combination of offset tracking and line-of-sight displacements derived from differential interferometry is discussed in [8]. While these references relate to glacier flow mapping, the seismic community often employs the multi-aperture interferometry (MAI) approach, which is claimed to provide more precise estimates of the along-track displacement than the noncoherent cross correlation of detected images [9], [10]. At this point, we note that MAI essentially corresponds to the previously developed spectral diversity (SD) or split-spectrum technique applied to the azimuth direction for precise estimation of mutual shifts [11], [12]. Despite different nomenclature, both approaches are based upon the generation of two looks in the Doppler spectrum and their associated interferograms. The differential phase is then directly related to the mis-registration. In this paper, we will therefore refer to the approach as SD, in agreement with its first application to image coregistration in the azimuth direction in [11] and with the techniques proposed for coregistration of TOPS data acquired over stationary scenes [6]. Based on SD and combined with differential interferometric SAR (D-InSAR) measurements in line-of-sight, three-dimensional (3-D) displacements were mapped, e.g., for the Bam earthquake and Kilauea volcano [3], [13]. 
Most of the reported investigations to-date have used stripmap SAR data. Even in a recent publication discussing the Sentinel-1 performance for seismic applications, the TOPS signal characteristics were disregarded and the analysis of the attainable performance was restricted to assessing the impact of the azimuth and range resolutions on the measurement accuracy of surface displacements [14]. The contribution of this paper is the in-depth analysis of relevant TOPS signal characteristics and a proposed processing methodology for making TOPS data usable for mapping nonstationary scenarios.

The paper is organized as follows: Section II reviews the TOPS azimuth signal characteristics and the resulting ambitious image coregistration requirements. It also summarizes the theoretical accuracy constraints of commonly used coregistration techniques, such as cross correlation and SD. Section III then discusses the implications for nonstationary scenarios with azimuth motion within the scene. For a stringent and a relaxed coregistration accuracy scenario, the size of the estimation window is evaluated. This is followed by the derivation of DEM accuracy requirements that ensure the unbiased estimation of motion-induced offsets. An adaptive azimuth common band filter is suggested to compensate for spectral decorrelation due to antenna beam steering and large azimuth surface displacement. Section IV discusses speckle tracking results for the Lambert glacier mapped by TerraSAR-X using the experimental TOPS acquisition mode. Section $\mathrm{V}$ presents a TOPS-adapted approach using SD for deriving improved motion estimates and a more reliable interferometric phase retrieval for D-InSAR applications, again with examples from the TerraSAR-X Lambert Glacier data. A discussion on the reinterpretation of the measured D-InSAR phases is included before the summary section of the paper.

\section{Requirements ReView And Theoretical ACCURACIES}

\section{A. TOPS Azimuth Signal Characteristics}

The special TOPS azimuth signal properties are a consequence of steering the azimuth antenna beam from aft to fore during burst acquisition. The Doppler spectrum of the signal as a function of the azimuth position within the burst is illustrated in Fig. 1. The first sketch relates to the raw data burst of a four subswath TOPS timeline, similar to the TerraSAR-X data used in this work. Its duration is about one quarter of the total burst length, the rest being used for illuminating the other three subswaths. Different targets along the burst are illuminated with a different instantaneous Doppler centroid $f_{D C}$, which is distinct from the overall mean Doppler centroid $\overline{f_{D C}}$, that is usually annotated in the image product as a polynomial function of slant range. However, in this work, $\overline{f_{D C}}=0$ is assumed for simplicity, being also a reasonable approximation due to the total zero Doppler steering of TerraSAR-X [15]. Since focused, singlelook complex (SLC) TOPS data are the input for all further interferometric signal analysis, it is more helpful to illustrate the azimuth dependent Doppler centroid in the time-frequency diagram of the burst image (see Fig. 1). It is given by

$$
f_{D C}\left(t_{a}\right)=\frac{k_{r o t} \cdot k_{a}}{k_{a}-k_{r o t}} \cdot t_{a}=k_{T} \cdot t_{a}
$$

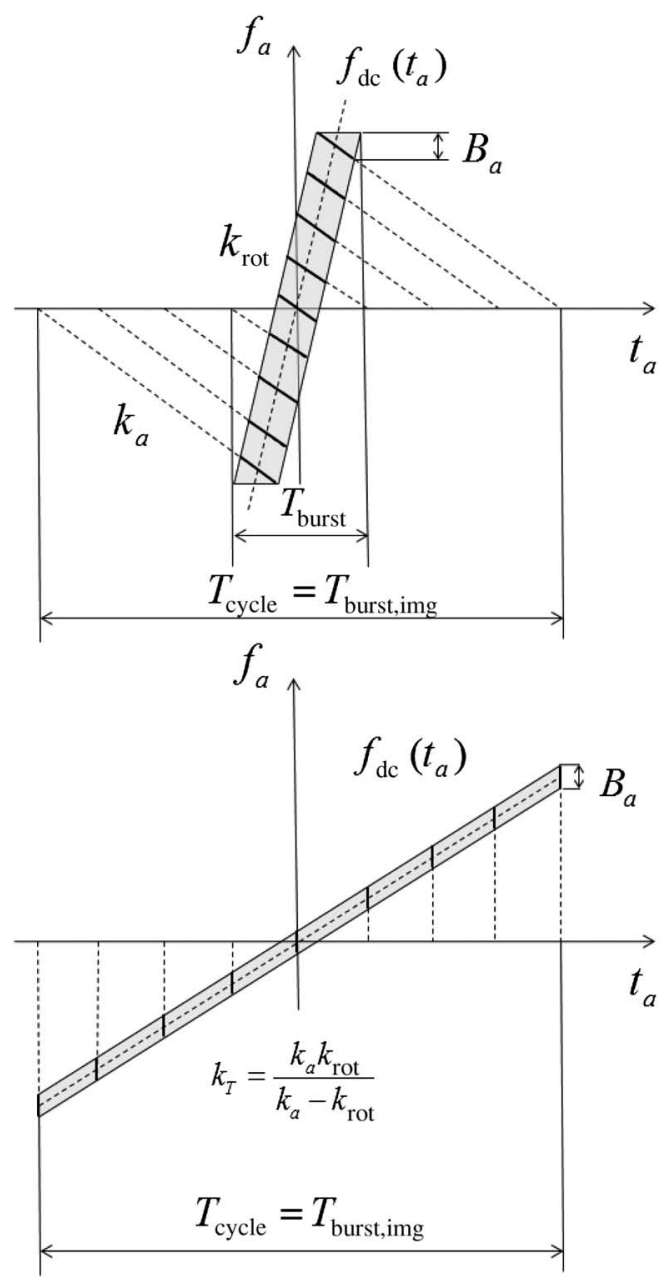

Fig. 1. Doppler spectrum of one burst of TOPS data: time-frequency diagram of raw data (top) and of image SLC data (bottom).

where $t_{a}=\left(x-x_{\text {mid }}\right) / v_{g}$ is the relative time within the burst, $x_{m i d}$ is the mid-azimuth burst position, and $v_{g}$ is the ground velocity. The Doppler rate $k_{a}$ is determined by the relative motion between sensor and target, whereas the rotation rate $k_{\text {rot }}$ is a function of the antenna beam steering in azimuth [5], [6]

$$
k_{a}=\frac{-2 v_{e f f}^{2}}{\lambda r}, \quad k_{r o t}=\frac{2 v_{e f f}^{2}}{\lambda r_{r o t}}=\frac{2 v_{s}}{\lambda} k_{\psi}
$$

where $v_{e f f}^{2}=v_{g} v_{s}$, with $v_{s}$ denoting the satellite velocity, $r_{r o t}$ the rotation range to the virtual rotation center of the acquisition (with negative sign as located behind the sensor), and $k_{\psi}$ the angular rotation rate of the beam. The azimuth variant Doppler centroid has several consequences for InSAR processing. While interferometric coregistration is discussed in Section II-B, we take this opportunity to point to the more general need for the so-called deramping operation of the Doppler spectrum. Any interpolation, upsampling or bandpass-filtering of the data in the azimuth direction requires base-banded data, unless spatially variant interpolation kernels are used. While the azimuth image sampling rate is only slightly higher than the target bandwidth $B_{a}$, the total excursion of Doppler frequencies will be many times larger (see Fig. 1). Deramping by the azimuth variant Doppler centroid of (1) prior to any of the aforementioned 
steps is therefore crucial. It corresponds to a quadratic phase function multiply along each burst. Reramping the Doppler spectrum after the corresponding operation is equally important to ensure phase preservation. For the particular case of interpolations (e.g., for interferometric coregistration), the deramping function needs to be interpolated in the same way as the resampling of the slave data, before it's conjugate is reapplied. Although not widely used, similar operations are also required for ScanSAR interferometry [16].

\section{B. Review of TOPS Coregistration Requirements}

Having summarized the Doppler dependency of targets along azimuth, it is important in the context of SAR interferometry to review the phase properties within the impulse response function (IRF). For non-zero Doppler centroids, a linear phase ramp-proportional to the Doppler offset-is present within the mainlobe of each target's IRF. For interferometric investigations in the presence of mis-registration, this may lead to local phase offsets [11]. In the case of TOPS mode InSAR pairs, due to the Doppler centroid variation, a constant azimuth misregistration $\Delta t$ leads to a linear phase ramp along each burst interferogram

$$
\phi_{b i a s}\left(t_{a}\right)=2 \pi f_{D C}\left(t_{a}\right) \Delta t=2 \pi k_{T} t_{a} \Delta t .
$$

The amount of linear phase excursion has been analyzed in detail in [6] and the methods to estimate accurately the constant azimuth coregistration offset between interferometric acquisitions have been developed and successfully tested with experimental TOPS data of TerraSAR-X. In particular, it was shown that the stringent coregistration requirement of better than 0.001 resolution cells can be achieved with estimates from the data, which limits the phase discontinuities at burst edges to less than $3^{\circ}$. This is considered equivalent to the refinement of the along-track orbital position knowledge to $1-2 \mathrm{~cm}$, improving the relative accuracy in state-of-the-art orbit determination between master and slave [17]. The analysis in [6], however, was focused on stationary scenes that are not affected by spatially variant displacements in the along-track direction.

\section{Theoretical Coregistration Estimation Accuracy}

Independent of the TOPS signal characteristics discussed above, the performance of coregistration estimation accuracy is derived in [12] from a theoretical viewpoint. The CramérRao (CR) bound in the estimation of the coregistration error is given by

$$
\sigma_{C R}=\sqrt{\frac{3}{2 N}} \cdot \frac{\sqrt{1-\gamma^{2}}}{\pi \gamma}
$$

in units of resolution cells, where $N$ is the number of independent samples averaged, which must take into account the oversampling and the weighting window in both dimensions, and $\gamma$ is the interferometric coherence. As was shown in [12], coherent cross correlation and coregistration by means of SD [11] can both achieve this bound, if properly implemented and parameterized. Here, we first analyze the achievable accuracy in estimating the mis-registration using ICC, i.e., on the basis of properly upsampled and detected amplitude signals as derived in $[18]^{1}$

$$
\sigma_{I C C}=\sqrt{\frac{3}{10 N}} \cdot \frac{\sqrt{2+5 \gamma^{2}-7 \gamma^{4}}}{\pi \gamma^{2}} .
$$

Following [12], the attainable coregistration accuracy using the $\mathrm{SD}$ method is given in terms of standard deviation of resolution cells

$$
\sigma_{S D}=\frac{\sqrt{2} \sigma_{l o o k}}{2 \pi} \cdot \frac{B_{a}}{\Delta f}
$$

where $B_{a}$ is the azimuth bandwidth of a single target, and $\sigma_{\text {look }}$ is the standard deviation of the interferometric phase of one of the looks

$$
\sigma_{\text {look }}=\sqrt{\frac{B_{a}}{b}} \cdot \sigma_{\text {int }}=\frac{1}{\sqrt{2 N}} \cdot \sqrt{\frac{B_{a}}{b}} \cdot \frac{\sqrt{1-\gamma^{2}}}{\gamma} .
$$

$\sigma_{\text {int }}$ is the phase standard deviation of the full-resolution interferogram, $b$ is the look bandwidth, and $\gamma$ is the interferometric coherence. As indicated in [12], when $b=B_{a} / 3, \Delta f=$ $B_{a}-b$, the SD solution approaches the CR bound given by (4) for large values of $N$.

For a stationary scene, the azimuth coregistration performance for experimental TerraSAR-X TOPS interferometric pairs was analyzed in [6]. It was shown that considering all samples within the burst, the stringent requirement of 0.00065 image samples can be achieved using the SD approach applied to the target bandwidth $B_{a}$. Furthermore, an extended SD (ESD) approach was suggested for the burst overlap regions to allow a more sensitive measurement on the basis of fewer samples.

\section{NONSTATIONARY COREgistration Estimates}

Based on the theoretical discussion of Section II, the accuracy limits are evaluated for a given estimation window size for the ICC according to (5) and for the SD according to (6). They are shown in Fig. 2 along with the burst edge requirement of a maximum $3^{\circ}$ phase discontinuity. The plots assume that the estimation window is square when converted into meters and projected onto ground

$$
N=N_{a z} N_{r g}=R_{s a m p} N_{a z}^{2} .
$$

Since the ratio of single-look azimuth and ground range resolutions $R_{\text {samp }}$ for TerraSAR-X TOPS data is approximately six (for Sentinel-1 four), more samples by the same factor are used in range than in azimuth. For SD, the required performance is achieved by using a square estimation window region, which corresponds to more than $150 \times 600$ full-resolution, independent azimuth $\times$ range samples for a coherence larger than 0.8 .

\footnotetext{
${ }^{1}$ Coherent cross correlation, working with complex-valued data, will not be applicable easily in the presence of a nonstationary scene exhibiting fringes as a result of surface movement.
} 

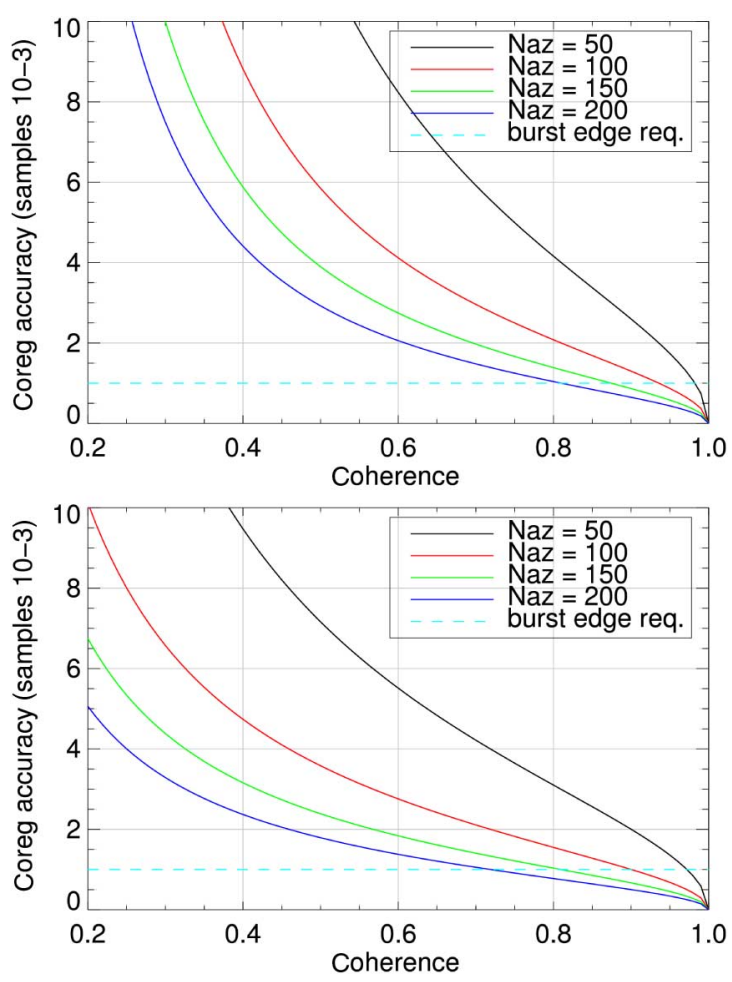

Fig. 2. Coregistration accuracy for different estimation window sizes $\left(R_{\text {samp }}=4\right)$. The performance is shown for ICC (top) and for the SD with spectral separation of $2 / 3$ of the azimuth bandwidth (bottom).

It is assumed that the true azimuth registration error is constant within this window. ICC is significantly worse than SD, in particular for lower coherence values. In either case, the requirement is relaxed with increasing azimuth distance from the burst edge [0.002 samples at one quarter (1/4) burst length offset from center, 0.004 samples at 1/8th burst length offset]. For the center of the burst, there is no specific coregistration requirement regarding phase artifacts. However, the usual 0.1 samples accuracy requirement applies to ensure unbiased coherence.

These evaluations show that the SD method is superior to ICC. This is confirmed by experimental results published in recent years [3], [10], [13], [14]. In order to ensure optimal coregistration performance for nonstationary scenes, the size of the estimation window needs to be adapted as a function of coherence and azimuth position within the burst. This is evaluated for the CR bound, as is appropriate for the SD method, and shown in the following two requirement scenarios.

\section{A. Stringent Requirement Case}

The stringent requirement corresponds to the one introduced for stationary scenes above, i.e., the systematic phase bias due to mis-registration shall be limited to $\pm 1.5^{\circ}$, independent of azimuth position within the burst and for all coherence values. As stated above, this is equivalent to a burst edge coregistration requirement of 0.00065 azimuth samples in the TerraSAR-X case. The required estimation window size strongly depends on the coherence and larger estimation windows are required at

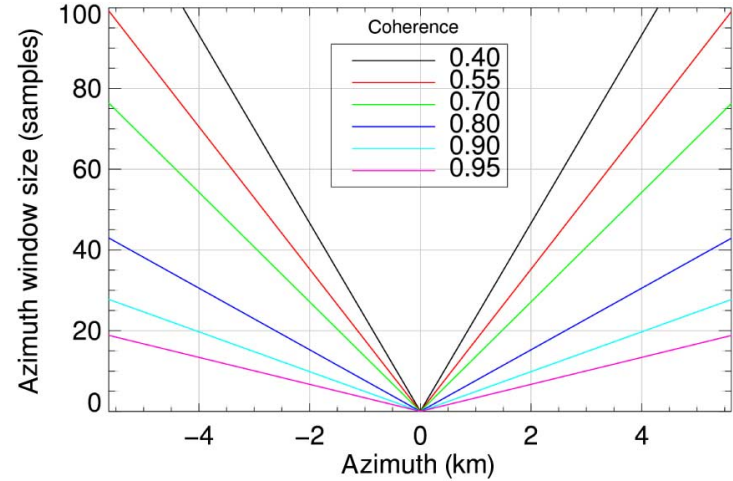

Fig. 3. Required estimation window azimuth size $N_{a z}$ for the stringent coregistration requirement case for TerraSAR-X TOPS data $\left(R_{\text {samp }}=6\right)$.

the burst edges. The number of independent samples (resolution cells) can be inferred from (4) and (1)

$$
N=\frac{3\left(1-\gamma^{2}\right)}{2 \pi^{2} \gamma^{2} \sigma_{C R}^{2}}=\frac{6\left(1-\gamma^{2}\right) f_{D C}^{2}\left(t_{a}\right)}{\gamma^{2} \phi_{\text {bias }, \text { max }}^{2}} d t^{2}
$$

where $\sigma_{C R}$ in this case corresponds to the required pixel coregistration accuracy $\Delta t=\sigma_{S D} d t, d t=d x / v_{g}$ is the azimuth image sampling, and $\phi_{\text {bias, } \max }$ is the maximum tolerated interferometric phase bias. A quantitative evaluation in terms of $N_{a z}=\sqrt{N / R_{\text {samp }}}$ is shown in Fig. 3 for the TerraSAR-X case with $R_{\text {samp }}=6$ and $\phi_{\text {bias } \text { max }}=1.5^{\circ}$. The results show that limiting the phase bias below a certain threshold has two consequences: 1) the window size needs to be adaptive with coherence and 2) an increasingly large estimation window is required toward the burst edge. Note that the TOPS azimuth burst size in the case of TerraSAR-X is only about 800 samples $(\approx 9 \mathrm{~km})$. Therefore, complying with this stringent requirement only seems sensible if the variations in the along-track surface displacements have spatial scales of several kilometers.

\section{B. Relaxed Requirement Case}

For small-scale spatial variations, which often occur as a result of seismic events or at the edges of moving glaciers and ice streams, considering relaxed requirements might be more appropriate. Indeed, one can argue that the stringent requirement on the phase bias induced by the limited coregistration accuracy can be relaxed to the order of magnitude of the interferometric phase standard deviation. This seems to be a reasonable suggestion, since perfect coregistration will not help once the scene gets decorrelated. In this case, the maximum phase bias due to mis-registration is assumed to be proportional to the interferometric phase standard deviation $\phi_{i f, s t d e v}$

$$
\phi_{\text {bias }, \text { max }}=k \cdot \phi_{i f, \text { stdev }}=\frac{k}{\sqrt{2 N_{L}}} \frac{\sqrt{1-\gamma^{2}}}{\gamma}
$$

with $N_{L}$ being the number of interferometric looks for the intended interferogram [1], [19]. Combining (10) with (9), the dependency on coherence cancels out

$$
N=\frac{12 N_{L} f_{D C}^{2}\left(t_{a}\right)}{k^{2}} d t^{2}
$$




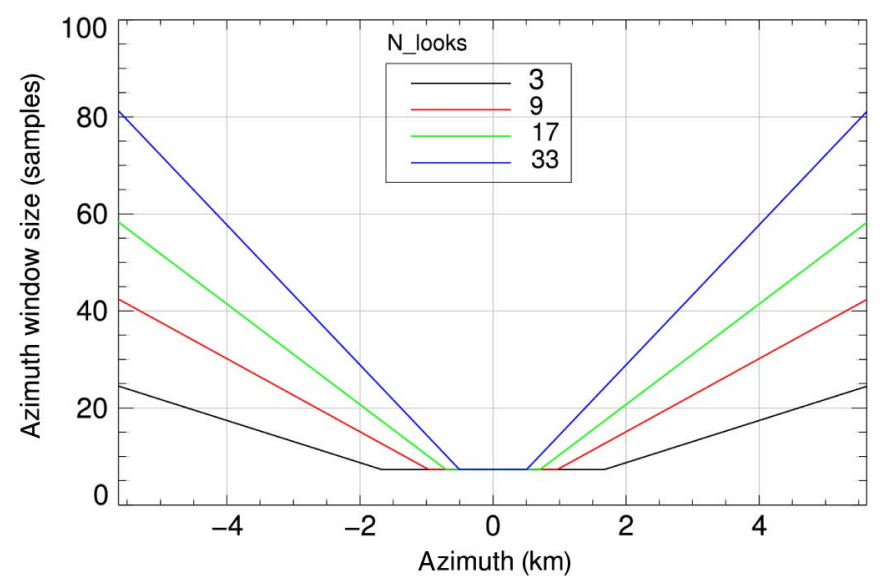

Fig. 4. Required estimation window azimuth size $N_{a z}$ for the relaxed coregistration requirement case with $k=0.5$, corresponding to a maximum phase bias due to azimuth mis-registration of half the interferometric phase standard deviation $\left(R_{\text {samp }}=6\right)$.

The arbitrary constant $k$ has been included to allow for consideration of a more stringent $(k<1)$ or of a more relaxed requirement $(k>1)$. The azimuth estimation window size can thus be parameterized by the number of interferometric looks, as displayed in Fig. 4 for the case $k=0.5$. At burst center, a minimum window size of $N_{a z}=7$ has been imposed to ensure a coregistration accuracy of 0.05 image samples for a coherence of $\gamma=0.4$. The necessary window size is seen to reduce considerably in comparison with the stringent requirement above, especially when considering low coherence areas and a reduced number of interferometric looks. In this case, a typical estimation window size for TerraSAR-X would be in the order of $360 \times 60$ ground range resolution cells, corresponding to an area on ground of approximately $800 \mathrm{~m} \times 800 \mathrm{~m}$ (about $420 \times 70$ image pixels due to oversampling). Note that this relaxed coregistration requirement may also reasonably be adopted for stationary scenes.

\section{DEM Error Induced Azimuth Mis-registration}

Since displacement estimates may become locally biased by systematic deficiencies in processing, this section addresses the residual azimuth mis-registration error introduced by computing the warping function for nominal interferometric coregistration using inaccurate terrain information. The sensitivity analysis equations were previously established in [20]. Due to the small orbital tube of TerraSAR-X and Sentinel-1 (diameters of $250 \mathrm{~m}$ and $100 \mathrm{~m}$, respectively, [4]), we use the approximate formula to evaluate the azimuth mis-registration error as a function of topographic height error $\Delta h$

$$
\Delta \text { pix } x_{\text {topo }}=\sin \alpha \cdot \cot \theta \cdot \Delta h / d x
$$

where $\alpha$ is the horizontal orbit crossing angle, $\theta$ is the look angle, and $d x$ is the TOPS image azimuth sampling in meters. $\alpha$ can also be expressed as a function of the along track baseline $B_{l}$ between master and slave acquisitions $B_{l}=r \sin \alpha \sin \theta$. The look angles for the full-performance TerraSAR-X and Sentinel- 1 data takes lie between $19^{\circ}-50^{\circ}$ and $20^{\circ}-45^{\circ}$, respectively, with $19^{\circ}$ being the worst case. Orbit crossing angles

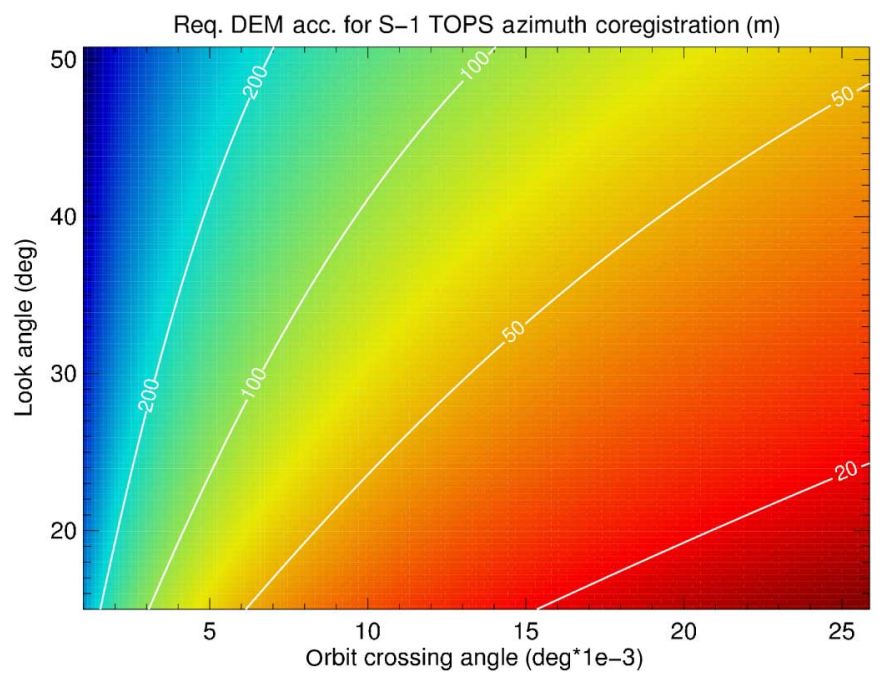

Fig. 5. Required DEM accuracy for precise azimuth coregistration of $1 \mathrm{~cm}$ (approximately 0.001 TOPS IW azimuth sample). Nominal Sentinel-1 look angles are between $20^{\circ}$ and $45^{\circ}$ and typical orbit crossing angles will be smaller than $2 \mathrm{e}-3$.

may vary considerably and would need to be computed for each interferometric pair in order to perform a realistic sensitivity analysis. A conservative value typical for ERS/ENVISAT, however, would be $0.025^{\circ}$, corresponding to an along-track baseline between $100 \mathrm{~m}$ and $300 \mathrm{~m}$. To ensure an azimuth mis-registration bias smaller than 0.0005 TOPS resolution cells ( 1 resolution cell corresponds to $20 \mathrm{~m}$ ) for the worst case parameters, the allowable topographic height error must not exceed $16 \mathrm{~m}$, which would correspond to a DTED-2 standard DEM (e.g., SRTM). By contrast, to ensure unbiased coherence, corresponding to the then much relaxed requirement of 0.1 resolution cells, the topographic sensitivity is about $1600 \mathrm{~m}$. Relaxed consideration of topography is also possible whenever the orbits become more parallel, up to the point where, for perfectly parallel orbits, the DEM has no influence upon azimuth coregistration offsets.

Finally, the topographic height knowledge requirement, as derived from (12) is plotted in Fig. 5 as a function of look angle $\theta$ and orbit crossing angle $\alpha$. The orbit crossing angles for the TerraSAR-X data takes of Lambert Glacier used in this study are less than $0.002^{\circ}$ and thus more than one order of magnitude lower than the conservative assumption of $0.025^{\circ}$. Therefore, for nominal azimuth coregistration purposes, it is sufficient to consider topographic information with an accuracy of about $100 \mathrm{~m}$.

\section{Adaptive Azimuth CBW Filter}

Common bandwidth (CBW) filtering is usually applied to compensate for different mean Doppler centroids and orbit crossing angles, thus minimizing the interferometric phase noise. The adaptive azimuth CBW filter for TOPS data discussed in this section is motivated by the fact that the Doppler variation in azimuth along with surface displacement in alongtrack causes additional spectral decorrelation between master and slave images. The amount of azimuth spectral shift depends 


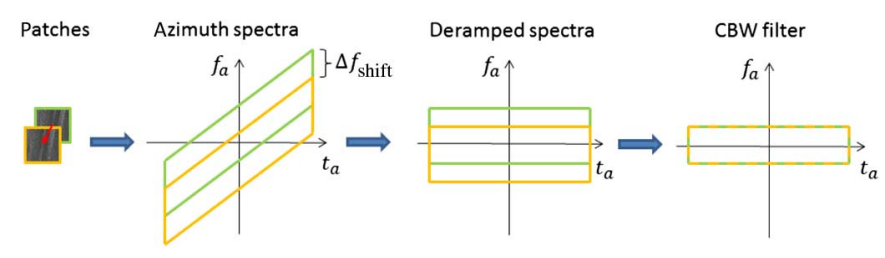

Fig. 6. Overview of the adaptive CBW filtering process, including azimuth time-frequency diagrams of master and slave patches at various stages of the processing.

linearly on the azimuth displacement and the individual processed Doppler centroids as follows:

$$
\Delta f_{\text {shift }}=k_{T} \cdot \Delta_{a z} / v_{g}+\overline{f_{D C}^{m}}-\overline{f_{D C}^{s}}+k_{T} \cdot \Delta t_{a c q}
$$

where $k_{T}$ denotes the total Doppler rate as introduced in Section II, $\Delta_{a z}$ denotes the azimuth displacement between the master and slave patches and $\overline{f_{D C}^{m}}$ and $\overline{f_{D C}^{s}}$ are the mean acquisition Doppler centroids for master and slave, respectively. The last-term in (13) corresponds to the contribution in terms of Doppler shift from the burst mis-synchronization of the data acquisition, given by $\Delta t_{a c q}$. Note that burst missynchronization can be properly accounted for by deramping the slave data using the master deramping function, provided that the relative shift $\Delta t_{a c q}$ has been corrected first. Furthermore, by filtering the master and slave images to retain only the common part of the Doppler spectra, the interferometric coherence can be improved. In the context of speckle tracking, this improvement can in turn make the ICC step more robust, leading to more accurate estimates.

The azimuth adaptive CBW filter is applied to pairs of patches taken from the master and slave SLCs, using displacement estimates of a first iteration to offset the locations of slave patches. The adopted implementation uses the same block size as the offset estimation itself and a 50\% block overlap to avoid artifacts at patch edges. Fig. 6 outlines the filtering process itself. It is important to deramp the master and slave images using the master deramping function to ensure that the resulting spectra are aligned before CBW filtering. To be precise, corresponding image regions in master and slave should be deramped in the same way, and it is important to note that these might not be at the same image locations due to local movement within the scene $\left(\Delta_{a z}\right)$. The deramping function for the slave is thus obtained by interpolating the master function using the displacement estimates already available to ensure that corresponding image contents are deramped in the same manner (as opposed to corresponding image locations). Once patches are deramped with spectra aligned as shown in the center timefrequency plot of Fig. 6, CBW filtering consists in transforming patches into the azimuth frequency domain and removing nonoverlapping regions of the spectra on the basis of the processed azimuth bandwidth and the spectral shift $\Delta f_{\text {shift }}$ computed from (13). This filtering operation is carried out after removing any azimuth spectral weighting applied by the processor for side-lobe suppression (a joint master and slave weighting function is reapplied after CBW filtering, considering the reduced bandwidth).
TABLE I

TERRASAR-X TOPS PARAMETERS FOR LAMBERT GLACIER

\begin{tabular}{c|c}
\hline \multicolumn{1}{|c||}{ Parameter } & Value (per subswath) \\
\hline Center frequency & $9.65 \mathrm{GHz}$ \\
Orbit height & $514 \mathrm{~km}$ \\
Acquisition dates 2012 & May 24 and June 4, 15, 26 \\
Azimuth resolution & $18.5 \mathrm{~m}$ \\
Ground range resolution & $\approx 3 \mathrm{~m}$ \\
Target azimuth bandwidth $\left(B_{a}\right)$ & $450 \mathrm{~Hz}$ \\
Total Doppler rate $\left(k_{T}\right)$ & $4857 / 4747 / 4609 / 4529 \mathrm{~Hz}^{2}$ \\
Azimuth burst size & $9503 / 9393 / 9013 / 9151 \mathrm{~m}$ \\
\hline
\end{tabular}

The effectiveness of the filter in practice depends on a combination of two factors: 1) displacements must be sufficiently large to cause non-negligible spectral decorrelation. For the TerraSAR-X datasets used in this study, total spectral decorrelation occurs at displacements of about $800 \mathrm{~m}$ and above. For azimuth filtering to have an appreciable impact, displacements between acquisitions must amount to a significant fraction of this distance. In consequence only very fast flowing glacier areas, like Jakobshawn in Greenland with around $10 \mathrm{~km} / \mathrm{year}$ (309 $\mathrm{m}$ for the 11 days repeat-cycle of TerraSAR-X) are expected to be affected [21]. 2) Temporal decorrelation plays a role, in that it also affects the region of CBW after filtering. Significant levels of temporal decorrelation mean that little interferometric coherence can be recovered in any way at all. This will be further discussed with the Lambert glacier example in Section IV.

\section{Speckle Tracking Results}

The results presented in this section concern the evaluation of the speckle tracking processing chain with respect to interferometric TerraSAR-X TOPS data pairs acquired on descending orbit over the Lambert glacier, Antarctica. The relevant parameters are summarized in Table I. The speckle tracking has been performed in a manner similar to [7].

Fig. 7 visualizes the estimated displacements, within the 11 day interval of the May and first June acquisitions, expressed in meters per day. The TOPS image consisting of 4 subswath in range (horizontal) and 12 azimuth bursts (vertical) is also shown, together with the estimated coherence after deterministic geometric coregistration and resampling to account for the estimated shifts. The fastest flowing parts of the Lambert glacier are estimated to move about $2 \mathrm{~m} /$ day and are located in the second subswath. Here, the displacements occur mainly in the along-track direction and correspond to 1.5 resolution cells. By comparing the results obtained with subsequent interferometric pairs, the accuracy of the glacier flow estimates is evaluated to be in the order of $4 \mathrm{~cm} /$ day, which is in agreement with other glacier tracking results reported for Radarsat-1 data [8].

The additional steps to be applied when working with TOPS data are the deramping before the upsampling of the complex bursts for detection and, when the estimation is carried out iteratively, the adaptive CBW filtering described in Section III-D. However, two factors may cause the adaptive CBW filtering to be ineffective, that are small surface displacements and temporal decorrelation. 1) Even the fastest observed azimuth velocity 

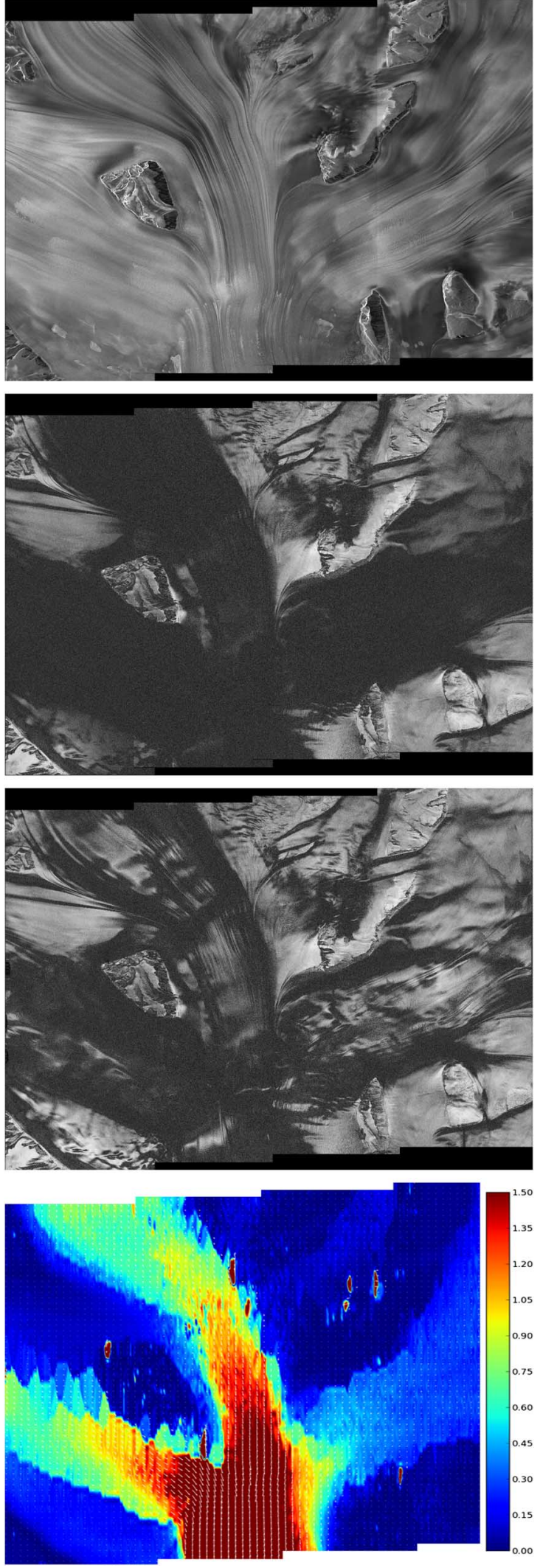

Fig. 7. TerraSAR-X image of the Lambert glacier, Antarctica. (Top) SAR magnitude, (middle top) coherence after geometric coregistration, (middle bottom) coherence after speckle tracking, and estimated 2-D surface displacement, (bottom) arrows indicating direction and magnitude of ice flow in meters per day.

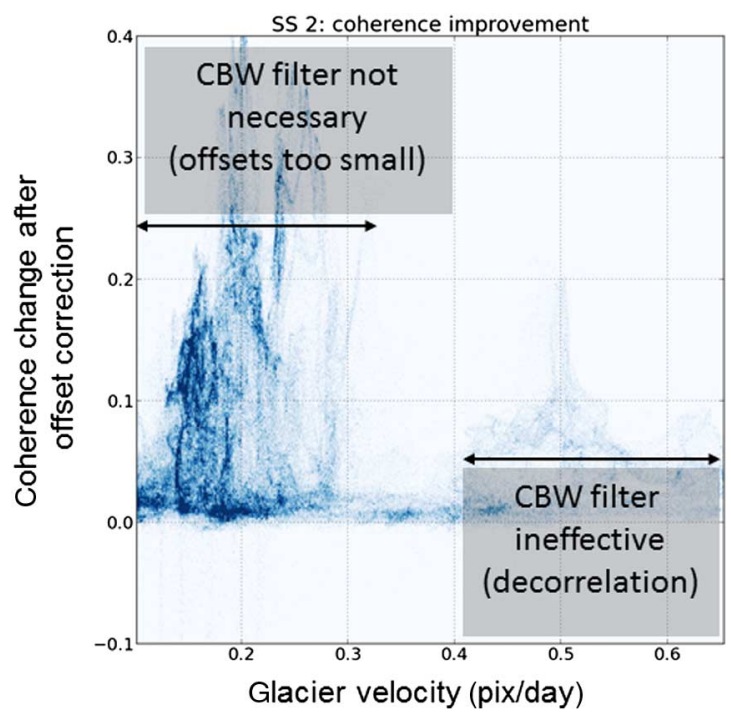

Fig. 8. Coherence improvements obtained for subswath 2 pixels, after correcting the 22-day interval slave SLC image using the estimated displacements as function of the magnitude of the applied correction.

of around $2 \mathrm{~m} /$ day corresponds, for the longest investigated temporal baseline of 22 days, to a spectral decorrelation of only around 5\%.2) Temporal decorrelation affects all parts of the spectrum and cannot be compensated for by filtering. Sufficient temporal decorrelation will in fact make speckle tracking ineffective all together, such that the measured azimuth shifts are rather the result of feature tracking, making CBW filtering irrelevant. The impact of temporal decorrelation is visible in Fig. 8, which depicts the coherence improvement after coregistration using the estimated range and azimuth offsets. Reliable displacement estimates on the glacier are identified as those for which the three investigated temporal baselines gave consistent offsets (within $0.2 \mathrm{~m} /$ day) and some motion is taking place $(1.0 \mathrm{~m} /$ day or greater). The scatter plot shows, for these reliable samples only, the coherence improvement obtained by correcting the slave image for the estimated displacement as a function of the displacement magnitude. A seemingly reasonable expectation would be that correcting large displacements (coregistration errors) would lead to correspondingly large improvements in coherence. For velocities below 0.3 pixels/day, this is indeed seen to be the case. For larger velocities, however, the gain in coherence quickly drops off, indicating higher levels of decorrelation: temporal decorrelation is more pronounced for parts of the glacier that move faster. A plausible explanation for this phenomenon is that fast-moving parts of the glacier may also experience more acceleration or rotation, both of which cause decorrelation of the SAR signal. Regardless of the actual physical reason, the plot explains why accounting for TOPS specific spectral shifts has not brought significant gains in this case.

\section{COREgistration FOR D-InSAR APPLICATIONS}

This section discusses the TOPS specific processing steps when D-InSAR measurements are intended for more precise estimates of displacements in the radar line-of-sight direction. 


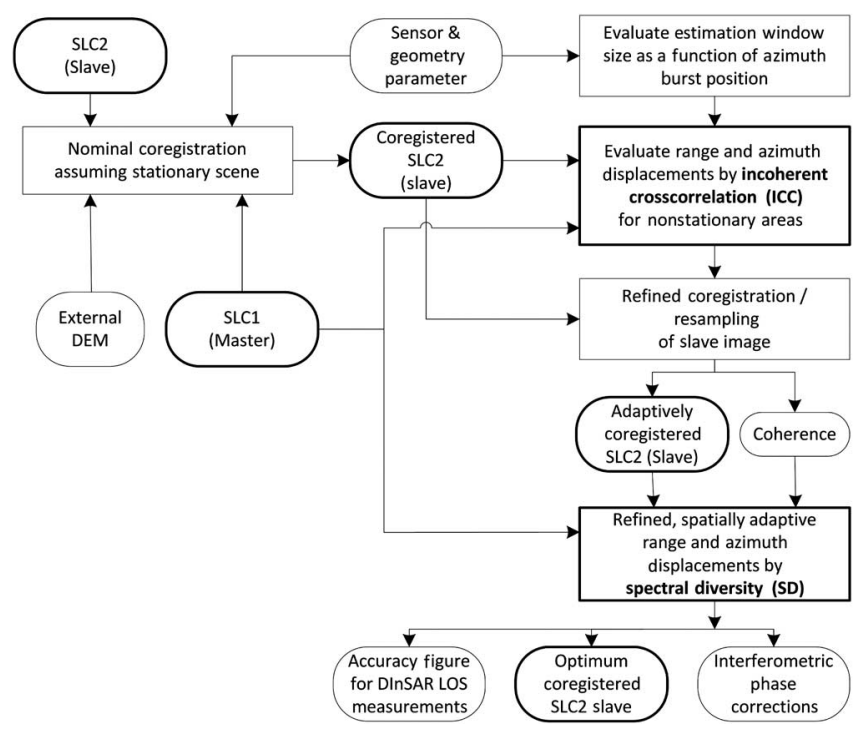

Fig. 9. Processing strategy for accurate coregistration of TOPS data for D-InSAR analysis in case of nonstationary scenes.

First, the required processing steps are discussed, followed by TerraSAR-X results from the Lambert glacier.

\section{A. Refined Adaptive Coregistration for TOPS Data Based on $S D$}

For adaptively correcting the phase bias induced by local azimuth offsets between the master and slave image, the following procedure is proposed (see also Fig. 9).

1) Evaluation of the estimation window size for coregistration as a function of azimuth position within the burst, according to Section III-B.

2) Resampling the slave image with nominal coregistration parameters derived from a DEM and assuming a stable scene, as outlined in Section II-A taking into account the TOPS specific signal properties.

3) Speckle tracking by ICC using a window size corresponding to a sample accuracy of at least 0.1 , as discussed in Section IV.

4) Resampling the nominally coregistered slave in range and azimuth according to the offsets obtained by speckle tracking.

5) Refining the estimation of azimuth offsets with SD (see details below).

6) Resampling the nominally coregistered slave image in range and azimuth to compensate for the refined offsets estimated with ICC and SD.

The SD estimation starts with the generation of two nonoverlapping azimuth spectral looks having a spectral separation of $\Delta f$ equal to $2 / 3$ of the common azimuth bandwidth. This process involves the following steps.

1) Basebanding/deramping of the full resolution master and slave SLC images by the azimuth-variant Doppler centroid function (see Section II).

2) Bandpass filtering, including the removal of the spectral weighting applied to the full resolution data.
3) Reramping the two complex valued looks (fore and aft looking).

4) Generation of the interferograms for both looks, followed by oversampling of the interferograms in both dimensions to avoid spectral aliasing in the subsequent steps. The oversampling might be traded against slight averaging of the individual interferograms, if a priori phase flattening is possible.

5) Estimation of differential complex-valued interferogram between looks and averaging considering the required window size discussed in Section III-B. Note that this step cancels out the topographic component and possible atmospheric phase bias for all intents and purposes, being nearly identical in the two interferograms due to quasi parallel orbits [6].

6) Estimation of the coregistration error $\Delta t=\phi_{\text {diff }} /$ $(2 \pi \Delta f), \phi_{\text {diff }}$ denoting the phase of the averaged differential interferogram, and $\Delta f$ the spectral separation between the SD looks.

In general, the main drawback of the SD technique is that the estimate is subject to phase wrapping errors. The maximum motion that can be measured unambiguously is given by [11]

$$
\Delta t_{\max }= \pm\left.\frac{1}{2 \Delta f}\right|_{\Delta f=2 / 3 B_{a}}= \pm \frac{3}{4 B_{a}} .
$$

In other words, as long as the azimuth shift is within \pm 0.75 resolution cells, it can be estimated unambiguously. In the presented approach, such difficulties are avoided because the ICC coregistration has already been applied, leading to sufficiently small residual mis-registration errors.

A computationally more efficient, but potentially less accurate, alternative to the last resampling step would be to compute the interferometric phase correction values within the burst, depending on the estimated azimuth offset measured by SD and the azimuth position within the burst, and apply them to the interferometric phase or to the coregistered slave obtained by means of ICC. At this point it is worthwhile reminding that the computed shifts (sum of ICC and SD estimates) might be interpreted as contributions to true line-of-sight measurements, as will be discussed in Section V-C.

To complement the algorithmic description of the processing chain above, theoretical considerations in previous sections can be combined into a figure of merit relating the coregistration uncertainty to the interferometric phase and thus to the inferred LOS displacement. Its derivation can take into account all relevant variations, such as coherence and azimuth position within the burst. From (1) and (4), the interferometric phase uncertainty due to the limited coregistration accuracy is given as

$$
\sigma_{\phi_{\text {bias }}}=2 \pi \cdot\left|f_{D C}\left(t_{0}\right)\right| \cdot \frac{d x}{v_{g}} \cdot \sqrt{\frac{3}{2 N}} \cdot \frac{\sqrt{1-\gamma^{2}}}{\pi \gamma} .
$$

If $N$ is determined based on the stringent requirement of eq. (9), the interferometric phase variance becomes independent on coherence, whereas if $N$ is estimated based on the relaxed requirement of eq. (11), it becomes independent of the azimuth position within the burst (except for the influence of the $N_{a z, \min }$ requirement). 


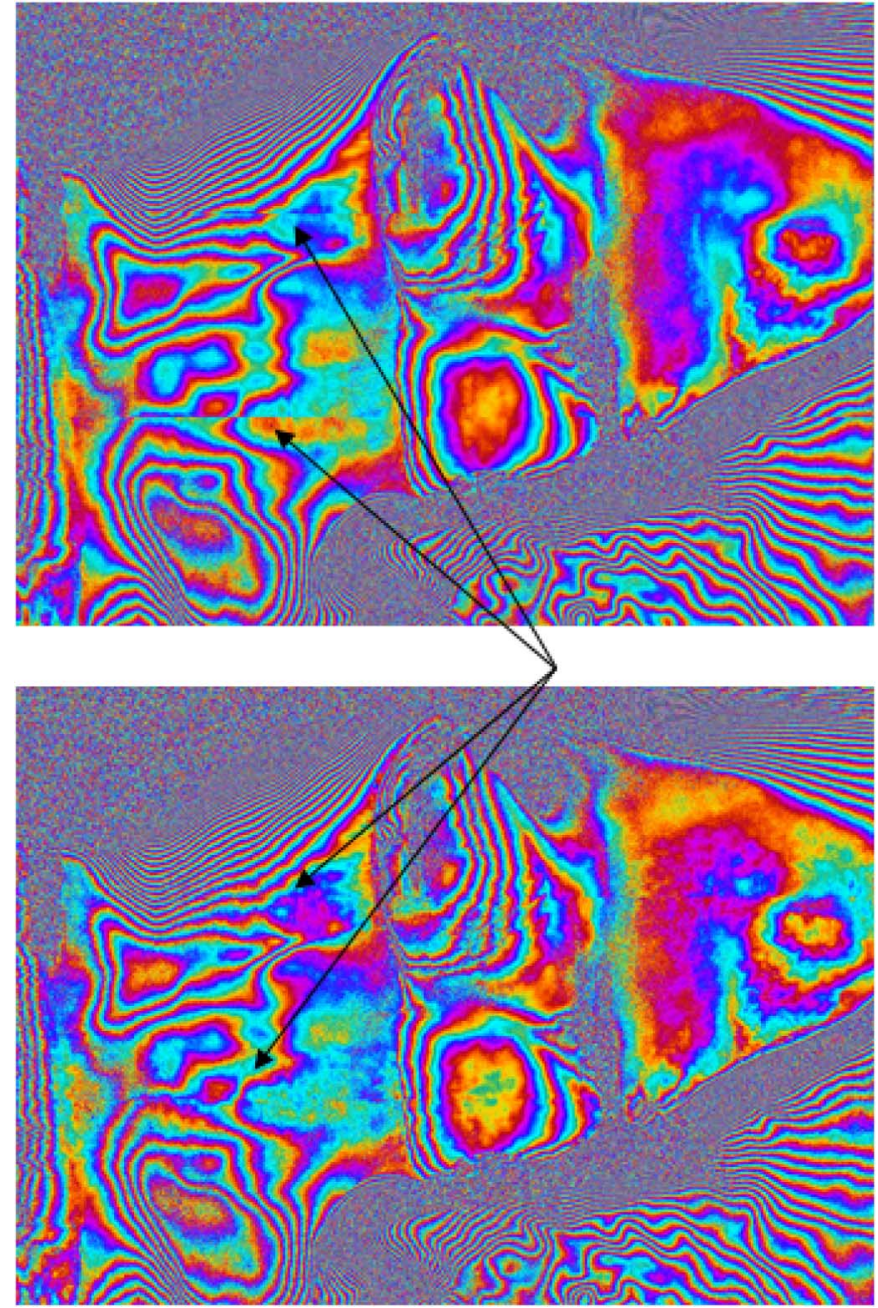

Fig. 10. TerraSAR-X TOPS interferometric phase of three bursts after coregistration with ICC/speckle tracking (top) and improved result after using SD (bottom). Phase discontinuities at burst edges are removed.

\section{B. Real Data Example}

The interferometric phase of the first three bursts of subswath 4 of the TerraSAR-X data pair of the Lambert glacier used in Section IV is shown in Fig. 10. The top image is obtained as a result of nominal coregistration (assuming a stationary scene) and subsequent speckle tracking by ICC to account for the glacier motion. No DEM was used for processing this data set. Therefore, the topographic phase and fringes due to glacier movement cannot be distinguished. Nevertheless, obvious phase discontinuities at burst edges clearly show that the coregistration by means of speckle tracking is not sufficiently accurate. The situation improves after SD has been used to refine the estimated azimuth shifts. Fig. 11 represents a more detailed analysis of this comparison for the third subswath. A coherence threshold of 0.4 was applied and invalid regions appear in black. The relaxed requirement according to (11) was used to determine the dimensions of the averaging window for the SD estimates, with $N_{L}=17$. A threshold of $N_{a z, \min }=36$ samples was adopted to comply with the coregistration requirement of 0.01 samples. According to Fig. 4, the estimation window size increases to 57 samples toward the burst edge.
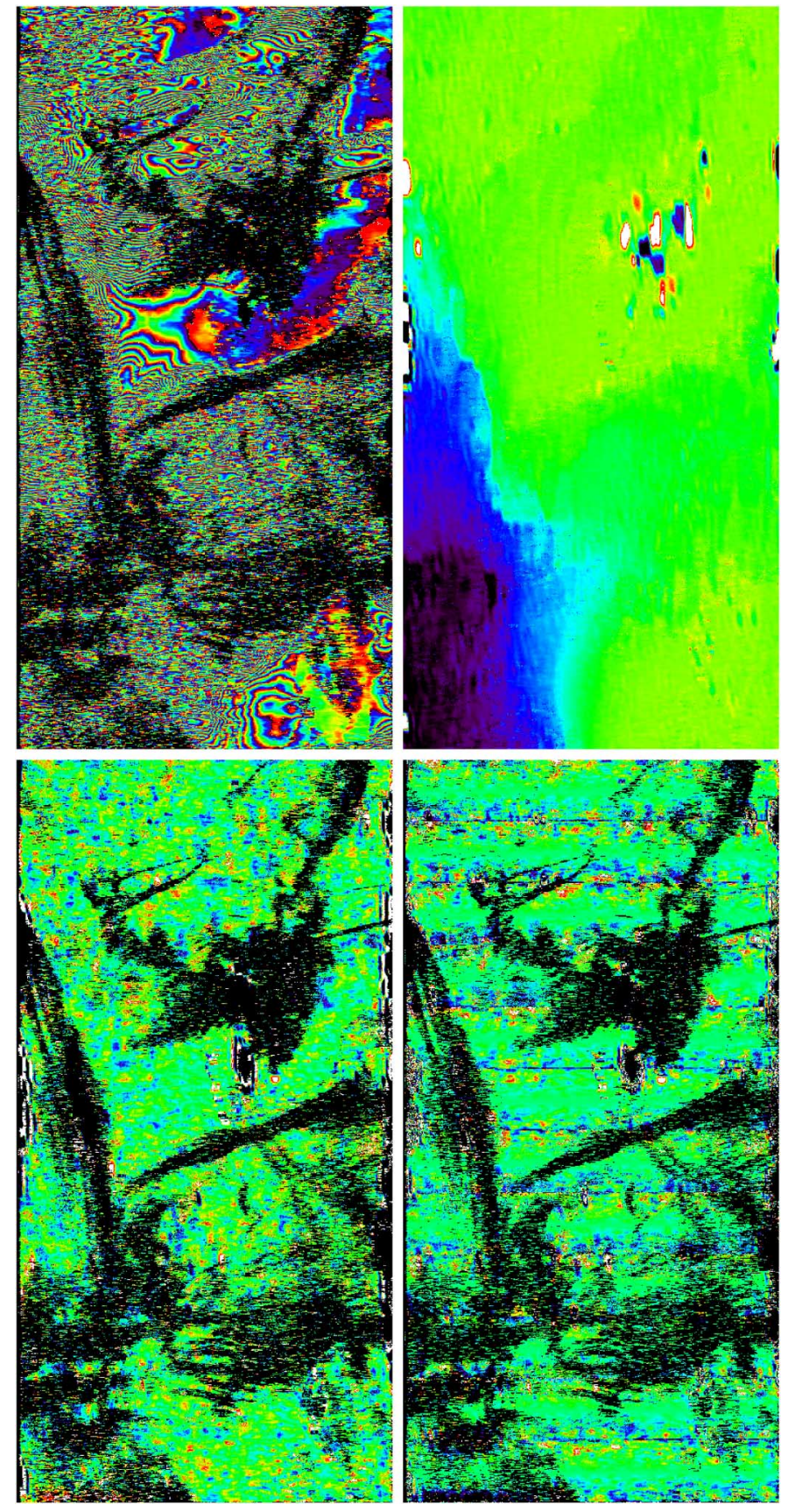

Fig. 11. Results for the third subswath: interferometric phase after flattening with DEM and coregistration with SD (top left), estimated azimuth offsets (scaled from $-20 \mathrm{~m}$ to $+10 \mathrm{~m}$ ) (top right) difference of local azimuth offsets (scaled \pm 0.1 resolution cells) (bottom left), and phase difference between same acquisition slave images coregistrered to the master by ICC and by ICC+SD (scaled $\pm \pi / 2$ ) (bottom right).

One can notice spatially localized differences in the offset estimates of ICC and SD in the order of up to 0.1 samples. This is most likely due to interpolation artifacts in the ICC values close to incoherent areas and at the burst edges. These are the locations where one would expect differences in the interferometric phase. These are best visualized when computing the differential phase between the coregistred slaves using: 1) the speckle tracking offsets only and 2) the refined estimates provided by SD. As expected, the differential phase is more 
sensitive to azimuth coregistration offset differences at the burst edges, whereas it cancels at the center of each burst, as can be easily recognized in Fig. 11 (bottom right). This analysis, in combination with Fig. 10, confirms the theoretical expectation that SD is more accurate than ICC. It also validates the proposed processing approach and demonstrates that D-InSAR measurements are possible with TOPS data, if properly handled.

\section{Phase Discontinuities Reinterpreted}

From the relaxed case discussed in Section III-B and used for the above analysis, it is clear that it is not possible to reach a sufficient accuracy in the coregistration considering the same number of samples for coregistration and interferometric phase averaging. As soon as the target is located at some distance from the burst center, the required window for azimuth shift estimation $(N)$ is definitely larger than the window $\left(N_{L}\right)$ used for estimating the InSAR phase.

This means that the two operations cannot be performed at the same scale. Still, it is possible to estimate and correct for (spatially) low-pass azimuth shift components, for which a large number of samples can be averaged. In a sense, this is the spirit of the ESD technique [6], which is able to correct for a rigid shift between the images, e.g., the shift caused by orbit timing inaccuracies. With this compensation, the residual bias affecting the interferometric phase will be negligible.

For long data takes, as foreseen to be acquired by Sentinel-1, it might be necessary to estimate not only a rigid shift but also a slowly varying component along azimuth. This estimate would collect all contributions, both those generated by the instrument/platform (i.e., clock drifts, orbit errors) and those that have a geophysical nature (e.g., solid Earth's tides). After a global or low-pass azimuth shift coregistration, the interferogram may still have phase contributions ascribable to local azimuth shifts and-locally-even clear phase discontinuities between bursts. However, such phase discontinuities are physical and should not be considered as a disturbance. They can be reinterpreted considering that in TOPS imaging, the line of sight geometry varies linearly with azimuth, and in particular, it jumps abruptly at burst edges [22]. Therefore, when surface motion does not occur in the zero-Doppler plane, there will be phase discontinuities if the line of sight changes abruptly, as occurs at burst edges. Even if the jumps could look aesthetically unpleasant, they should be treated as information and not as disturbance. Some applications could even benefit from having two distinct lines of sight in regions of burst overlap, which can give additional constraints to the geophysical deformation models.

Here, it maybe worth noting that such discontinuities are present also in ScanSAR interferograms, where they are just less visible due to the reduced Doppler excursion. They will also be not visible in TOPS interferograms for small motions or motions limited to the zero-Doppler plane, like subsidence.

Phase unwrapping across burst edges, however, might be a serious issue in case of local azimuth motion. We propose here two ways on how to proceed. One could apply a local coregistration in order to eliminate phase jumps (and recover coherence). The interferometric phase quality will be limited after local coregistration, but the elimination of the discontinuities will make phase unwrapping easier. After phase unwrapping, the phase that was implicitly subtracted in the coregistration step can be added back to the interferogram, recovering the full interferometric quality for the unwrapped phase. This is the rationale for the approach described in Section V-A. Alternatively, one could unwrap the interferogram burst by burst and finally recover the absolute phase with radargrammetry applied to each burst.

\section{CONCLUSION}

This paper has addressed the issues involved in the processing of nonstationary interferometric SAR acquisitions in TOPS mode, which is the main operational mode on Sentinel-1. The suggested methodology can be applied not only for glacier flow estimation but also for measuring of slip motion in seismically active areas. For the purpose of this study, interferometric TOPS pairs were acquired with the TerraSAR-X sensor over the Lambert glacier, Antarctica. These datasets were used as the basis for demonstrating the estimation of the glacier velocity field using speckle tracking by means of ICC. A conventional patch-based cross correlation approach, as commonly applied to stripmap data, was shown to be suitable also for TOPS data, provided that the azimuth-variant Doppler centroid is considered when oversampling the master and slave patches before detection. On the other hand, the adaptive azimuth CBW filter developed herein specifically for TOPS mode data pairs has shown an almost negligible performance improvement, mainly due to the fact that regions of high glacier velocity are usually also subject to considerable geometrical decorrelation due to rotation or acceleration. The proposed filtering approach could, however, become relevant in scenarios where large azimuth displacements occur without the aforementioned geometrical and temporal effects and an improvement in the interferometric coherence would lead to more reliable motion estimates. The probably more relevant contribution of this work is related to the specific precautions when using D-InSAR techniques, especially if the motion is small. Due to the large azimuth variation in the Doppler centroid along each TOPS burst, special care needs to be taken to avoid artifacts in the differential interferometric phase, especially at burst edges. A methodology has been proposed to estimate more accurately the 2-D surface displacement map based on SD, thus avoiding phase discontinuities at burst edges. The key result is the definition of an estimation window size as a function of the azimuth position within the burst, but independent of coherence. This results in a critical trade-off between phase accuracy and spatial resolution of the estimation. Depending on the specifics of the surface motion to be estimated and the interferometric coherence, small scale details are potentially lost, unless the interferometric phase is reinterpreted taking into account the variable azimuth beam angle dependent line-of-sight along each burst. Cross-checks with different pairs of the investigated glacier scenario have shown encouraging and consistent 
results, but the presence of large accelerations and/or rotations in the scene make the interpretability of the differential phase difficult. In this sense, the analysis of 3-D surface motion, as caused by crustal deformation after seismic events, would be more suited in order to fully evaluate the proposed methodology. Suitable interferometric pairs covering co-seismic events are expected to be available more often once systematic and routine Sentinel-1 TOPS acquisitions become operational.

\section{REFERENCES}

[1] P. A. Rosen et al., "Synthetic aperture radar interferometry," Proc. IEEE, vol. 88, no. 3, pp. 333-382, Mar. 2000.

[2] E. Rignot, J. Mouginot, and B. Scheuchl, "Ice flow of the antarctic ice sheet," Science, vol. 333, no. 6048, pp. 1427-1430, Aug. 2011, doi: $10.1126 /$ science. 1208336 .

[3] E. Erten, A. Reigber, and O. Hellwich, "Generation of three-dimensional deformation maps from InSAR data using spectral diversity techniques," ISPRS J. Photogramm. Remote Sens., vol. 65, pp. 388-394, Jun. 2010.

[4] R. Torres et al., "GMES Sentinel-1 mission," Remote Sens. Environ., vol. 120, pp. 9-24, 2012.

[5] F. De Zan and A. Monti Guarnieri, "TOPSAR: Terrain observation by progressive scans," IEEE Trans. Geosci. Remote Sens., vol. 44, no. 9, pp. 2352-2360, Sep. 2006.

[6] P. Prats-Iraola, R. Scheiber, L. Marotti, S. Wollstadt, and A. Reigber, "TOPS interferometry with TerraSAR-X," IEEE Trans. Geosci. Remote Sens., vol. 50, no. 8, pp. 3179-3188, Aug. 2012.

[7] T. Strozzi, A. Luckman, T. Murray, U. Wegmüller, and C. L. Werner, "Glacier motion estimation using SAR offset-tracking procedures," IEEE Trans. Geosci. Remote Sens., vol. 40, no. 11, pp. 2384-2391, Nov. 2002.

[8] H. Liu, Z. Zhao, and K. Jezek, "Synergistic fusion of interferometric and speckle-tracking methods for deriving surface velocity from interferometric SAR data," IEEE Geosci. Remote Sens. Lett., vol. 4, no. 1, p. 102-106, Jan. 2007.

[9] N. B. D. Bechor and H. A. Zebker, "Measuring two-dimensional movements using a single InSAR pair," Geophys. Res. Lett., vol. 33, no. 16, pp. L1611-1-L16 311-5, Aug. 2006, doi: 10.1029/2006GL026 883.

[10] H.-S. Jung, J.-S. Won, and S.-W. Kim, "An improvement of the performance of multiple-aperture SAR interferometry (MAI)," IEEE Trans. Geosci. Remote Sens., vol. 47, no. 8, p. 2859-2869, Aug. 2009.

[11] R. Scheiber and A. Moreira, "Coregistration of interferometric SAR images using spectral diversity," IEEE Trans. Geosci. Remote Sens., vol. 38, no. 5, pp. 2179-2191, Jul. 2000.

[12] R. Bamler and M. Eineder, "Accuracy of differential shift estimation by correlation and split-bandwidth interferometry for wideband and Delta-k SAR systems," IEEE Geosci. Remote Sens. Lett., vol. 2, no. 2, pp. 151155 , Apr. 2005.

[13] H.-S. Jung, Z. Lu, J. S. Won, M. P. Poland, and A. Miklius, "Mapping three-dimensional surface deformation by combining multiple-aperture interferometry and conventional interferometry: Application to the june 2007 eruption of Kilauea volcano, Hawaii," IEEE Geosci. Remote Sens. Lett., vol. 8, no. 1, p. 34-38, Jan. 2011.

[14] H.-S. Jung, Z. Lu, and L. Zhang, "Feasibility of along-track displacement measurement from Sentinel-1 interferometric wide-swath mode," IEEE Trans. Geosci. Remote Sens., vol. 51, no. 1, pp. 573-578, Jan. 2013.

[15] H. Fiedler, E. Börner, J. Mittermayer, and G. Krieger, "Total Zero Doppler Steering-A new method for minimizing the Doppler centroid,' IEEE Geosci. Remote Sens. Lett., vol. 2, no. 2, pp. 141-145, Apr. 2005.

[16] J. Holzner and R. Bamler, "Burst-mode and ScanSAR interferometry," IEEE Trans. Geosci. Remote Sens., vol. 40, no. 9, pp. 1917-1934, Sep. 2002.

[17] Y. Yoon, M. Eineder, N. Yague-Martinez, and O. Montenbruck, "TerraSAR-X precise trajectory estimation and quality assessment," IEEE Trans. Geosci. Remote Sens., vol. 47, no. 6, p. 1859-1868, Jun. 2009.

[18] F. D. Zan, "Accuracy of incoherent speckle tracking for circular Gaussian signals," IEEE Geosci. Remote Sens. Lett., vol. 11, no. 1, pp. 264-267, Jan. 2014.

[19] E. Rodriguez and J. M. Martin, "Theory and design of interferometric synthetic aperture radars," IEE Proc. Inst. Elect. Eng. F, vol. 139, no. 2, pp. 147-159, Apr. 1992.

[20] E. Sansosti, P. Berardino, M. Manunta, F. Serafino, and G. Fornaro, "Geometrical SAR image registration," IEEE Trans. Geosci. Remote Sens., vol. 44, no. 10, pp. 2861-2870, Oct. 2007.
[21] I. Joughin, B. Smith, D. Shean, and D. Floricioiu, "Brief communication: Further summer speedup of Jakobshavn isbrae," Cryosphere, vol. 8, pp. 209-214, 2014, doi: 10.5194/tc-8-209-2014.

[22] F. De Zan, P. Prats, R. Scheiber, and A. Rucci, "Interferometry with TOPS: coregistration and azimuth shifts," in Proc. EUSAR, Berlin, Germany, Jun. 3-5, 2014, pp. 1-4.

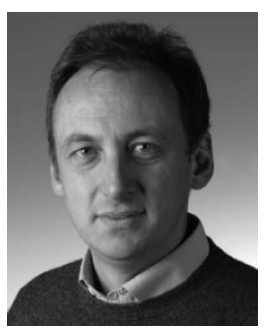

Rolf Scheiber received the Diploma degree from the Technical University of Munich, Munich, Germany, in 1994, and the Ph.D. degree from the University of Karlsruhe, Karlsruhe, Germany, in 2003, both in electrical engineering.

Since 1994, he has been with the Microwaves and Radar Institute, German Aerospace Center (DLR), Wessling, Germany, where he developed the operational high-precision interferometric SAR processor for its E-SAR airborne sensor. Since 2001, he has been heading the SAR Signal Processing group in the SAR Technology department. His research interests include algorithm development for high-resolution air- and space-borne SAR focusing, SAR interferometry, differential SAR interferometry, SAR tomography, as well as radio sounding algorithms and applications.

Dr. Scheiber was awarded as coauthor with the 1996 GRSS Transactions Prize Paper Award for the contribution Chirp Scaling Algorithm for Air- and Space-borne SAR Data Processing in Stripmap and ScanSAR Imaging Modes in 1997.

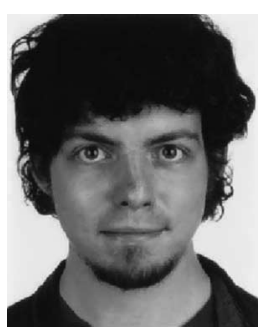

Marc Jäger was born in Karlsruhe, Germany, in 1979. He received the B.A. (Hons.) degree in computer science from the University of Cambridge, Cambridge, U.K., in 2001, the M.Sc. degree (with distinction) in digital signal and image processing from DeMontfort University, Leicester, U.K., in 2003, and is currently pursuing the Ph.D. degree in the automatic extraction of semantic information from polarimetric and polarimetric interferometric SAR data at Computer Vision and Remote Sensing Group, Berlin University of Technology,

Berlin, Germany.

Currently, he is with the German Aerospace Center (DLR), Oberpfaffenhofen, Germany, primarily on the polarimetric and interferometric processing of airborne SAR data. His research interests include the unsupervised classification and hierarchical segmentation of SAR data, anisotropic diffusion, and graphical models for inference in object recognition problems.

Mr. Jäger received a Student Paper Award in the Sixth European Conference on Synthetic Aperture Radar (EUSAR 2006).

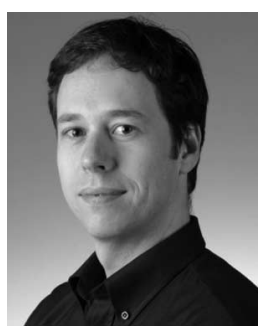

Pau Prats-Iraola (S'03-M'06-SM'13) was born in Madrid, Spain, in 1977. He received the Ingeniero degree in telecommunication engineering and the Ph.D. degree from the Universitat Politècnica de Catalunya (UPC), Barcelona, Spain, in 2001 and 2006, respectively.

In 2001, he was a Research Assistant with the Institute of Geomatics, Barcelona, Spain. In 2002, he was with the Department of Signal Theory and Communications, UPC, where he worked in the field of airborne repeat-pass interferometry and airborne differential SAR interferometry. From December 2002 to August 2006, he was an Assistant Professor with the Department of Telecommunications and Systems Engineering, Universitat Autònoma de Barcelona, Barcelona, Spain. In 2006, he joined the Microwaves and Radar Institute, German Aerospace Center (DLR), Wessling, Germany, where, since 2009, he has been the Head of the Multimodal Algorithms Group. His research interests include highresolution airborne/spaceborne SAR processing and SAR interferometry.

Dr. Prats-Iraola was the recipient of the first prize of the Student Paper Competition of the 2005 IEEE INTERNATIONAL GEOSCIENCE AND REMOTE SENSING S YMPOSIUM (IGARSS), Seoul, Korea. In 2012, he was awarded with the DLR Science Award. 


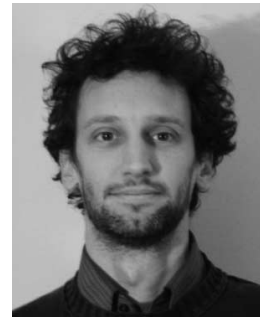

Francesco De Zan received the Master's degree in telecommunication engineering and the Ph.D. degree in synthetic aperture radar (SAR) interferometry with stacks from Politecnico di Milano, Milan, Italy, in 2004 and 2008, respectively.

During his studies, he has collaborated with T.R.E., Milan, Italy, on persistent scatterer interferometry and with ESA for the Sentinel-1 mission. In 2007, he visited the Stanford Exploration Project at Stanford University, Stanford, CA, USA, for 6 months. Since 2008, he has been with the Microwaves and Radar Institute of German Aerospace Center (DLR), Oberpfaffenhofen, Germany. There he has worked on the calibration of the TanDEM-X interferometer; he has been involved in studies for future SAR missions like BIOMASS, Tandem-L, OSCM, SAOCOM-CS, and Sentinel-1. His research interests include modeling, performance analysis and optimum processing for SAR interferometry and radargrammetry.

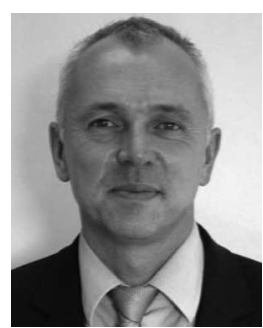

Dirk Geudtner received the M.S. degree in geophysics from the Technical University "Mining Academy" Freiberg, Freiberg, Germany, in 1991, and the $\mathrm{Ph} . \mathrm{D}$. degree in synthetic aperture radar (SAR) interferometry from the University of Stuttgart, Stuttgart, Germany, in 1995.

In 1991, he joined the German Aerospace Center (DLR), Oberpfaffenhofen, Germany, where he worked as a SAR Scientist on the development of new methods for satellite SAR interferometry (In- SAR) data processing and analysis. After joining the German Research Center for Geosciences (GFZ) in 1994, he was responsible for the development of geoscientific applications of satellite InSAR techniques. From 1995 to 1997 , he was a Visiting Scientist with the Canada Centre for Remote Sensing (CCRS), where he worked on differential InSAR for monitoring glacier and ice motion. In 1997, he joined the Microwaves and Radar Institute, DLR, where he developed InSAR calibration concepts for the X-SAR system on the Space Shuttle Radar Topography Mission (SRTM). During SRTM operations, he was the Lead Engineer for the in-orbit X-SAR interferometric antenna beam alignment at the National Aeronautics and Space Administrations Payload Operations Control Center, Houston, TX, USA. With a research grant from the German Humboldt-Foundation, between 2000 and 2002, he was a Visiting Scientist at CCRS, where he worked on new methods for the interferometric processing of Canadas RADARSAT-1 data and for the polarimetric calibration of RADARSAT-2 data. During a 6-year assignment to the Canadian Space Agency (CSA) between 2002 and 2008, he worked as Key SAR Scientist/Engineer on feasibility studies for future satellite SAR missions focusing particularly on the RADARSAT Constellation Mission (RCM) of CSA. For the RCM project, he was entrusted with the lead of the external Canadian SAR Expert Image Quality and Science Advisory Group. After returning back to DLR in 2008, he was responsible for the TerraSAR-X2 feasibility study and the analysis of methods for orbital baseline calibration for the TanDEM-X mission. From 2009 to 2011, he was a Scientific Advisor (Seconded National Expert) with the European Commissions Global Monitoring for Environment and Security (GMES) Bureau, where he supported the analysis of the GMES space component and was also responsible for the implementation of the GMES marine monitoring service. Since March 2011, he has been the Head of the Sentinel-1 Mission and Systems Section, European Space Agency, Noordwijk, The Netherlands. In this role, he is responsible for the compliance of the SAR system performance with user/science requirements, the end-to-end SAR system calibration, and the initiation and technical supervision of SAR system and application studies. 